\title{
Micro-drilled optical fiber for enhanced laser strain sensors
}

\author{
R. A. Perez-Herrera ${ }^{* a}$, M. Bravo Acha ${ }^{a}$, P. Roldan-Varona ${ }^{b, c}$, D. Leandro ${ }^{a}$, L. Rodriguez Cobo ${ }^{c}$, \\ J. M. Lopez-Higuera ${ }^{b, c, d}$, M. Lopez-Amo ${ }^{a}$ \\ ${ }^{a}$ Dept. of Electrical Electronic and Communication Engineering and Institute of Smart Cities (ISC), \\ Public University of Navarra, 31006 Pamplona, Spain; ${ }^{b}$ Photonics Engineering Group, University of \\ Cantabria, 39005 Santander, Spain; ' CIBER-bbn, Instituto de Salud Carlos III, 28029 Madrid, Spain; \\ 'Instituto de Investigación Sanitaria Valdecilla (IDIVAL), 39005 Cantabria, Spain
}

\begin{abstract}
In this work, we present an experimental measurement of temperature and strain sensitivities of a micro-drilled optical fiber (MDOF). The MDOF consisted of a quasi-randomly distributed reflector along a single mode fiber (SMF). A fiber cavity laser based on MDOF was experimentally studied, attaining a single-wavelength laser emission centered at $1568.6 \mathrm{~nm}$. The output power level obtained from this single-laser oscillation when pumped at $140 \mathrm{~mW}$ was around $9.6 \mathrm{dBm}$, and an optical signal to noise ratio (OSNR) of around $45 \mathrm{~dB}$ was measured. Although temperature sensitivities of fiber Bragg gratings used as sensors are similar to our MDOF, strain sensitivity is enhanced around one order of magnitude when the MDOF was used.
\end{abstract}

Keywords: fiber Bragg grating, fiber cavity laser, micro-drilled optical fiber, optical fiber sensor.

\section{INTRODUCTION}

Fiber optic lasers have been used since the 90s of the last century as sensor systems [1]. Fiber lasers have many advantages in the field of fiber optic sensors, such as high dynamic ranges and good levels of optical power, among others. In these lasers, transducers such as Fiber Bragg Gratings can act as the mirrors of the laser cavity. With them, lasers sensors can be realized with a short cavity [2-5], or having a cavity of tens of kilometers [6-7]. Recently, a new family of fiber lasers, that use a distributed cavity, have entered the field of fiber sensors [8,9]. These are based on a random feedback mechanism, supported by backscattering. An efficient gain for them can be achieved in several different ways. The conventional method is to use Raman amplification along several kilometers of fiber. This strategy is convenient for remote sensors applications [10]. However, sometimes short-cavity lasers are needed for sensing [11]. Nowadays new cavities have been developed in order to achieve short distributed mirrors for fiber lasers [12-13]. In this case, usual Er-doped fiber based amplification can be used, leading to short cavity laser structures [14]. The distributed lasing structures show improved features such as a modeless behavior, high output power stability or the possibility of being internally modulated, among others.

In this paper we evaluate the performance of a quasi-randomly distributed reflector written into a single-mode fiber as a sensing mirror in a fiber laser. With this mirror it is possible to develop a short-cavity fiber optic laser sensor which presents a higher strain sensitivity than regular FBG transducers. The distributed reflector consists of a micro-drilled single-mode fiber with a pseudo-random pitch. The reflector was fabricated using a femtosecond (fs) laser micromachining system that provided an enhancement in the inhomogeneity of the refractive index of the fiber, offering an increase in distributed scattering.

\section{EXPERIMENTAL SETUP}

\subsection{Inscription process}

For drilling the optical fiber, a standard transversal inscription setup was selected. A commercial femtosecond fiber laser chirped pulse amplifier (FLCPA; CALMAR Laser, Inc., USA) was used to generate pulses that were focused through a NA $=0.5$ objective lens (to converge at the sample which was placed on a nano-resolution motorized stage (Aerotech, Inc., USA). The stage was translated in a single direction parallel to the fiber length during execution of the micro-drilling inscription, following a process similar to the one previously used in [14]. The femtosecond laser pulses 
were centered at a wavelength of $1030 \mathrm{~nm}$; the pulse duration was $370 \mathrm{fs}$ and pulse energy was $0.47-0.9 \mu \mathrm{J}$; the writing speed was $0.1 \mathrm{~mm} / \mathrm{s}$. The pulse repetition rate was randomly modulated in order to inscribe the spots on the microwire with spatial periods in the range of $0-10 \mu \mathrm{m}$.

Figure 1 shows a photograph of the quasi-randomly distributed reflector along the single mode optical fiber. As may be seen in this figure, the randomness of the inscribed structured was achieved by randomly modulating the pulse repetition rate at every update (which corresponds to $100 \mathrm{~ms}$ approximately). Therefore, a quasi-random physically inscribed structure was achieved, with a varying envelope period of tenths of micrometers; the period was maintained for several tenths of micrometers and then randomly altered. The direct random inscription was not possible because the repetition rate of the femtosecond laser could not be simultaneously modulated at the same rate as the pulse inscription speed. Instead, quasi-randomly spaced spots were achieved, with random variations in spatial periods between groups of pulses, but a constant period within each envelope.

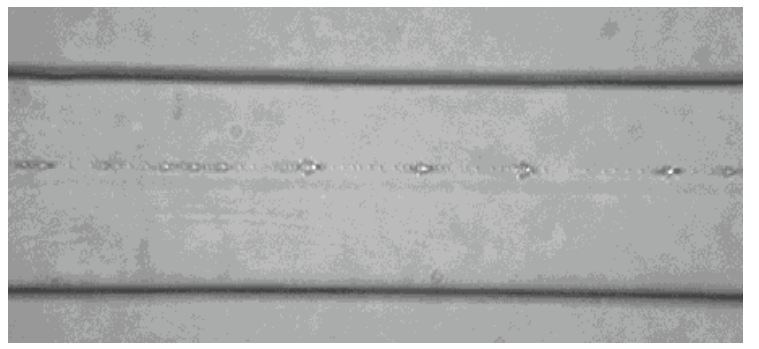

Figure 1. Photograph of the quasi-randomly distributed reflector along the SMF.

\subsection{Experimental set up}

Figure 2 shows a schematic diagram of the experimental setup. Here, a 980/1550 nm wavelength division multiplexer (WDM) injects the pump laser centered at $976 \mathrm{~nm}$ into the linear cavity fiber laser. The gain medium was located at the common port of the WDM and consists of $4 \mathrm{~m}$ of erbium-doped fiber (EDF). This EDF was type I25(980/125), with peak core absorption ranges from 7.7 to $9.4 \mathrm{~dB} / \mathrm{m}$ at $1531 \mathrm{~nm}$. This type of EDF is suitable for telecommunications Cband amplifiers due to its core composition optimized for erbium-dopes fiber amplifiers (EDFASs) in dense wavelength division multiplexing (DWDM) networks. The linear cavity of the laser ends at an optical circulator in which ports 3 and 1 are connected, acting as a fiber loop mirror (FLM). The recirculating signal travels through the 1500 -nm port of the WDM to an optical coupler where $20 \%$ of the signal was directing into an optical spectrum analyzer (OSA) with a resolution of $0.01 \mathrm{~nm}$ to monitor the spectrum, and $80 \%$ enters the micro-drilled fiber (MDF).

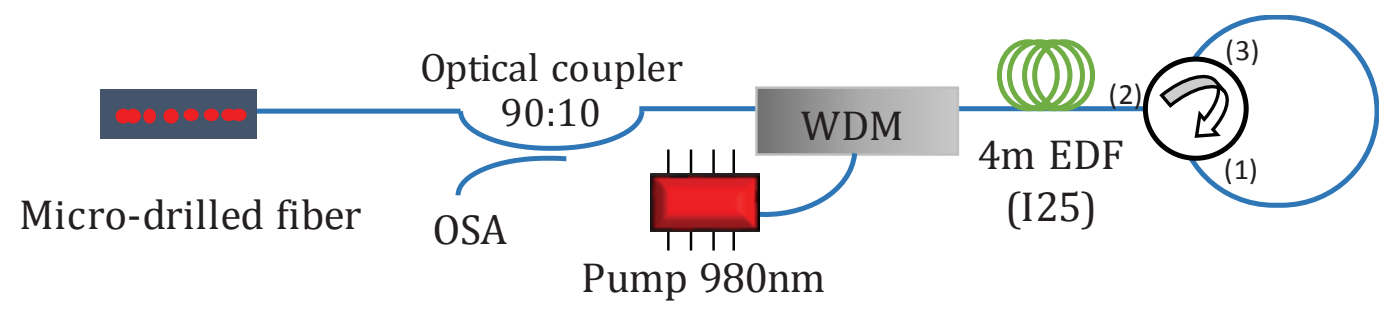

Figure 2. Schematic diagram of the experimental linear short-cavity fiber laser setup, in which the micro-perforated fiber was used to reflect an amplified signal.

\section{RESULTS}

A single-wavelength laser centered at $1568.6 \mathrm{~nm}$ was achieved employing the micro-drilled optical fiber. The output power level obtained from this single-laser oscillation when pumped at $140 \mathrm{~mW}$ was around $-9.6 \mathrm{dBm}$, and an OSNR of around $45 \mathrm{~dB}$ was measured as Fig. 3 illustrates. This structure was characterized as strain sensor at room temperature and also as temperature sensor when no strain is applied.

The temperature induced wavelength shift was characterized in order to determinate its sensitivity. This characterization was carried out by using a climatic chamber in the range of $25^{\circ} \mathrm{C}$ to $100^{\circ} \mathrm{C}$ and taking samples each $2^{\circ} \mathrm{C}$. As can be seen 
in Fig 4, the center wavelength shift for the laser centered at $1568.6 \mathrm{~nm}$ presents a clear linear behavior (the mean square error is equal to 0.9986 ) and a temperature sensitivity of about $10.2 \mathrm{pm} /{ }^{\circ} \mathrm{C}$ was measured. This attained value was close to the typical value for temperature-induced Bragg wavelength shift in silica fibers operating at $1550 \mathrm{~nm}$, which is around $11 \mathrm{pm} /{ }^{\circ} \mathrm{C}[15]$.

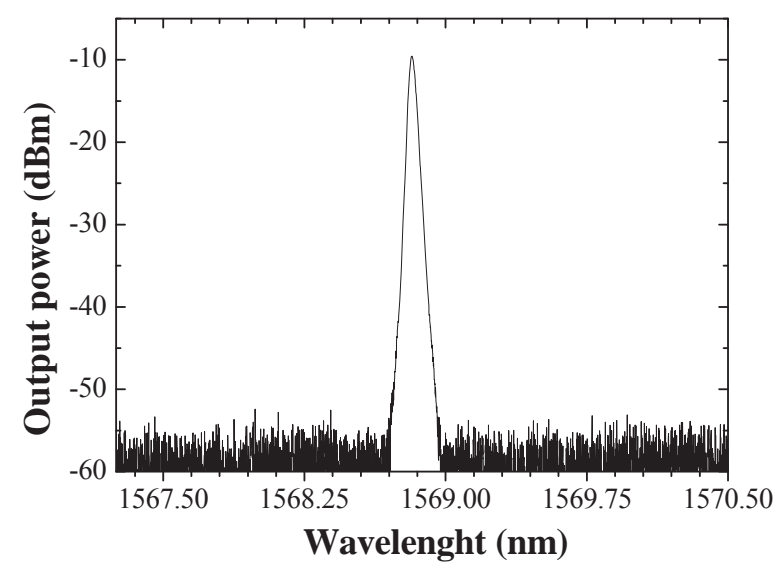

Figure 3. Output spectra of the short-linear-cavity fiber laser with MDF pumped by a $980-\mathrm{nm}$ laser at $140 \mathrm{~mW}$.

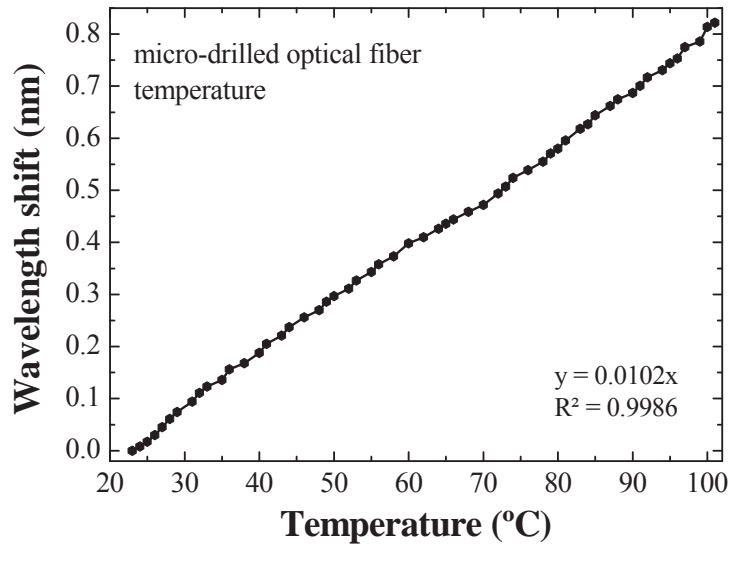

Figure 4. Wavelength shift as a function of temperature change.

Next, the sensor head based on MDOF was glued to two stress inducing plates (SP) motionless in order to evaluate the wavelength shift produced by straining it. The micro-displacements applied to the SP could be controlled by a highprecision motorized stage in order to modify their central-wavelength emission. A characterization of its microdeformation of 29 steps with $3.15 \mu \varepsilon$ per step was carried out.

Figure 5 shows the spectral shift of central emission wavelength when the structure is subjected to strain variations. The result shows a very linear response (the mean square error is almost equal to 1) with a slope sensitivity as good as 11.6 $\mathrm{pm} / \mu \varepsilon$. This value, when comparing with the typical value for strain induced Bragg wavelength shift, that is approximately $1.2 \mathrm{pm} / \mu \varepsilon[15,16]$, presents an enhancement of about one order of magnitude which represents a significant improvement in strain sensitivity.

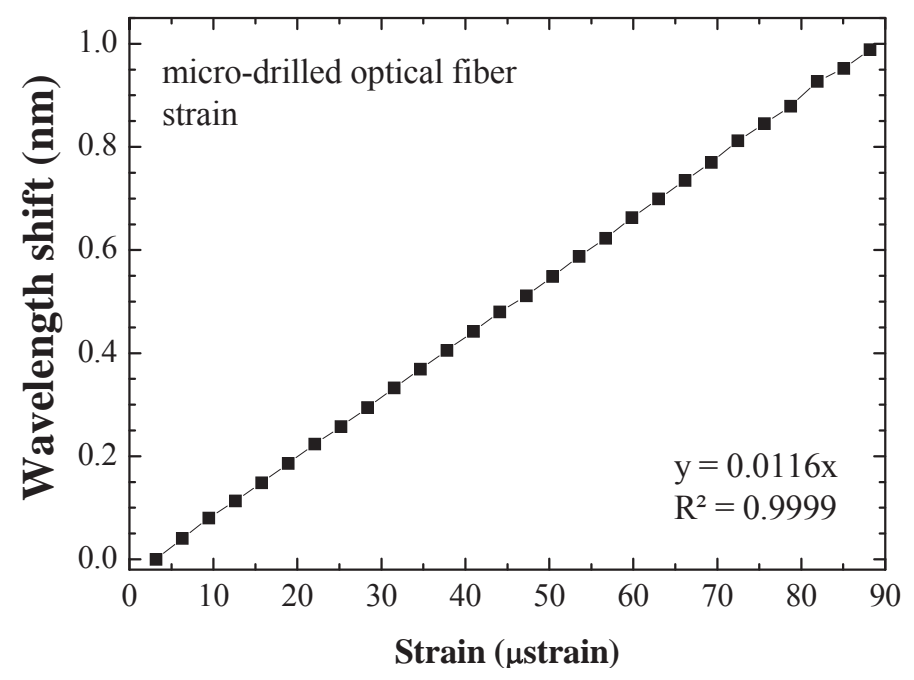

Figure 5. Wavelength shift as a function of strain change. 


\section{CONCLUSIONS}

In this work, a new fiber laser sensing system has been experimentally demonstrated for temperature and strain measurements. The laser incorporates a new MDOF reflector, fabricated by using a femtosecond laser micromachining system, consisted of a quasi-randomly distributed reflector along a single mode fiber (SMF). Single-wavelength laser emission at $1568.6 \mathrm{~nm}$ with an output power of about $-9.5 \mathrm{dBm}$ and an optical signal-to-noise ratio (OSNR) greater than $45 \mathrm{~dB}$ was measured. Although temperature sensitivities achieved were similar to FBGs ones, strain sensitivity enhanced around one order of magnitude in comparison with FBG sensors.

\section{ACKNOWLEDGMENT}

This work was supported by the Spanish AEI TEC2016-76021-C2, FEDER Funds, and the European Union's Horizon 2020 research and innovation programme under the Marie Skłodowska-Curie grant agreement No 838143.

\section{REFERENCES}

[1] A. D. Kersey and W. W. Morey, "Multi-element Bragg-grating based fibre-laser strain sensor," in Electronics Letters, 29(11), 964-966 (1993).

[2] L. Talaverano, S. Abad, S. Jarabo, and M. Lopez-Amo, "Multiwavelength fiber laser sources with Bragggrating sensor multiplexing capability," J. Lightwave Technol. 19(4), 553-558 (2001).

[3] Qi Wu, Yoji Okabe, and Junqiang Sun, "Investigation of dynamic properties of erbium fiber laser for ultrasonic sensing," Opt. Express 22, 8405-8419 (2014)

[4] O. Hadeler, M. Ibsen, and M. N. Zervas, "Distributed-feedback fiber laser sensor for simultaneous strain and temperature measurements operating in the radio-frequency domain," Appl. Opt. 40, 3169-3175 (2001)

[5] S. Avino, A. Giorgini and G. Gagliardi "Fiber-Optic Cavities for Physical and Chemical Sensing" The Open Optics Journal, 7, (Suppl-1, M8) 128-140 (2013)

[6] M Fernandez-Vallejo, S Díaz,, R A Perez-Herrera, D Passaro, S Selleri, M A Quintela, J M López Higuera and M Lopez-Amo "Resilient long-distance sensor system using a multiwavelength Raman laser" Measurement Science and Technology, 21, 094017 (5pp) (2010)

[7] D. Leandro, A. Ullan, A. Loayssa, et. al "Remote $(155 \mathrm{~km})$ Fiber Bragg Grating interrogation technique combining Raman, Brillouin, and Erbium gain in a fiber laser," IEEE Photon. techn. letters, 23, 623-627 (2011)

[8] O Frazão, C Correia. J L Santos et. al "Raman fibre Bragg-grating laser sensor with cooperative Rayleigh scattering for strain-temperature measurement" Measurement Science and Technology 20 (4), 045203 (2009)

[9] A. M. R. Pinto, M. Lopez-Amo, J. Kobelke and K. Schuster, "Temperature Fiber Laser Sensor Based on a Hybrid Cavity and a Random Mirror," in Journal of Lightwave Technology, 30(8), 1168-1172 (2012)

[10] V. DeMiguel-Soto, D. Leandro, and M. Lopez-Amo, "Ultra-long (290 km) remote interrogation sensor network based on a random distributed feedback fiber laser," Opt. Express 26, 27189-27200 (2018)

[11] Y. Shen, Y. Qiu, B. Wu, W. Zhao, S. Chen, T. Sun, and K. T. V. Grattan "Short cavity single frequency fiber laser for in-situ sensing applications over a wide temperature range," Optics Express 15, 363-370 (2007)

[12]X. Wang, D. Chen, H. Li, S. H. E. Lijuan, and Q. Wu, "Random fiber laser based on artificially controlled backscattering fibers," Appl. Opt. 57(2), 258-262 (2018).

[13]X. Wang, D. Chen, H. Li, and Q. Wu, "Artificially controlled backscattering in single mode fibers based on femtosecond laser fabricated reflectors," Opt. Commun. 413, 224-229 (2018).

[14] R. A. Perez-Herrera, D. Pallarés-Aldeiturriaga, A. Júdez, L. Rodriguez Cobo, M. Lopez-Amo, et. al, "Optical fiber lasers assisted by microdrilled optical fiber tapers," Opt. Lett. 44, 2669-2672 (2019)

[15] L.S. Grattan, B.T. Meggit, [Optical Fiber Sensor Technology: Devices and Technology], Vol. 2 Optoelectronics, Imaging and Sensing, Springer Science \& Business Media, 2012

[16] C. E.Campanella, A. Cuccovillo, C. Campanella, A. Yurt, V. Passaro, "Fibre Bragg Grating Based Strain Sensors: Review of Technology and Applications," Sensors, 18(9), 3115, (2018) 